\title{
First Cold Powering Test of REBCO Roebel Wound Coil for the EuCARD2 Future Magnet Development Project
}

Glyn A. Kirby, Jeroen van Nugteren, H. Bajas, V. Benda, A. Ballarino, M. Bajko, Luca Bottura, K. Broekens, M. Canale, A. Chiuchiolo, L. Gentini, N. Peray, Juan Carlos Perez, Gijs de Rijk, Adriaan Rijllart, Lucio Rossi, J. Murtomaeki, Jacky Mazet, Francois-Olivier Pincot, Giovanni Volpini, Maria Durante, P. Fazilleau, Clement Lorin, A. Stenvall, Wilfried Goldacker, Anna Kario, and Alexander Usoskin

(Invited Paper)

\begin{abstract}
EuCARD-2 is a project partly supported by FP7European Commission aiming at exploring accelerator magnet technology for $20 \mathrm{~T}$ dipole operating field. The EuCARD-2 collaboration is liaising with similar programs for high field magnets in the USA and Japan. EuCARD-2 focuses, through the workpackage 10 "Future magnets," on the development of a $10 \mathrm{kA}$-class superconducting, high current density cable suitable for accelerator magnets, for a $5 \mathrm{~T}$ stand-alone dipole of $\mathbf{4 0} \mathrm{mm}$ bore and about $1 \mathrm{~m}$ length. After standalone testing, the magnet will possibly be inserted in a large bore background dipole, to be tested at a peak field up to $18 \mathrm{~T}$. This paper starts by reporting on a few of the highlight simulations that demonstrate the progress made in predicting: dynamic current distribution and influence on field quality, complex quench propagation between tapes, and minimum quench energy in the multitape cable. The multiphysics output importantly helps predicting quench signals and guides the development of the novel early detection systems. Knowing current position within individual tapes of each cable we present stress distribution throughout the coils. We report on the development of the mechanical component and assembly processes selected for Feather-M2 the $5 \mathrm{~T}$ EuCARD2 magnet. We describe the CERN variable temperature flowing helium cold gas test system. We describe the parallel integration of the FPGA early quench detection system, using pickup
\end{abstract}

Manuscript received August 31, 2016; accepted January 6, 2017. Date of publication January 26, 2017; date of current version March 1, 2017. This work was supported by the European Commission under the FP7 Research Infrastructures project EuCARD-2, Grant agreement no. 312453.

G. A. Kirby, J. van Nugteren, H. Bajas, V. Benda, A. Ballarino, M. Bajko, L. Bottura, K. Broekens, M. Canale, A. Chiuchiolo, L. Gentini, N. Peray, J. C. Perez, G. de Rijk, A. Rijllart, L. Rossi, J. Murtomaeki, J. Mazet, and F.-O. Pincot are with the CERN, Geneva CH-1211, Switzerland (e-mail: Glyn.Kirby@ cern.ch; jeroen.van.nugteren@cern.ch; luca.bottura@cern.ch; Juan.Carlos. Perez@cern.ch; Gijs.derijk@cern.ch; Adriaan.Rijllart@cern.ch; Lucio.Rossi@ cern.ch; Jacky.Mazet@cern.ch; Francois-Olivier.Pincot@cern.ch).

G. Volpini is with the Istituto Nazionale di Fisica Nucleare Milano and CERN, Segrate 20090, Italy (e-mail: giovanni.volpini@mi.infn.it).

M. Durante, P. Fazilleau, and C. Lorin are with the CEA, Gif-surYvette 91191, France (e-mail: maria.durante @ cea.fr; philippe.fazilleau @ cea.fr; clement.lorin@gmail.com).

A. Stenvall is with the Tampere University of Technology (TUT) Korkeakoulunkatu 10, FI-33720 Tampere, Finland (e-mail: antti.stenvall@tut.fi).

W. Goldacker and A. Kario are with the Karlsruhe Institute of Technology, Eggenstein-Leopoldshafen 76344, Germany (e-mail: wilfried.Goldacker@ kit.edu; anna.kario@kit.edu).

A. Usoskin is with the Bruker HTS GmbH, Alzenau 63755, Germany (e-mail: alexander.usoskin@bruker.com).

Color versions of one or more of the figures in this paper are available online at http://ieeexplore.ieee.org.

Digital Object Identifier 10.1109/TASC.2017.2653204 coils and temperature sensors, alongside the standard CERN magnet quench detection system using voltage taps. Finally we report on the first cold tests of the REBCO $10 \mathrm{kA}$ class Roebel subscale coil named Feather-M0.

Index Terms-Superconducting magnets, accelerators magnets, high-temperature-superconductors, EuCARD-2, future magnets, HTS, Roebel cable, cryogenic systems, metal 3-D printing.

\section{INTRODUCTION}

$\mathbf{P}$ OST-LARGE Hadron Collider (LHC) energy-frontier collider development has started. Triggered by the first conceptual study for a high energy LHC (HE-LHC), based on 20 T collider quality dipole magnets [1]-[3], CERN has launched a collaboration aimed at exploring the use of high temperature superconductor (HTS) (the only material viable for field in excess of 15-16 T) for accelerator magnets.

EuCARD2 program work package 10 [4] has chosen to develop Roebel cable based on REBCO tape and the first magnets will be cold tested during 2016-2017. The EuCARD2 project is achieving its main goal with the development, manufacture and delivery of long lengths of multi strand HTS Roebel cable, now under way and being wound into test magnet coils. The aligned block accelerator type magnet named Feather-M2 [5] (see Fig. 1) is under construction at CERN Geneva and an alternative, Cosine Theta coil design is under construction at CEA-Saclay Paris. The detailed design for both magnets are complete and dummy coil winding with Roebel cable has started for both magnets: the 5 T magnet Feather-M2, and the Cosine-Theta magnet. Four short cable lengths approximately $6 \mathrm{~m}$ have been delivered and are planned to be used in a sequence of subscale coil windings (called Feather-M0) which will be cold tested. The first length of Robel cable was assembled with tape from Bruker (member of the EuCARD2 consortium). This cable was wound into a coil. Initial test results are presented later in this paper. Groundbreaking progress has been made in the areas of: cable production, magnetic simulation, assembly techniques and processes.

\section{REBCO CABLE \& MAGNETS Design Simulations}

During the course of the EuCARD2 program from 2013 many papers have been published which cover in detail the computer 


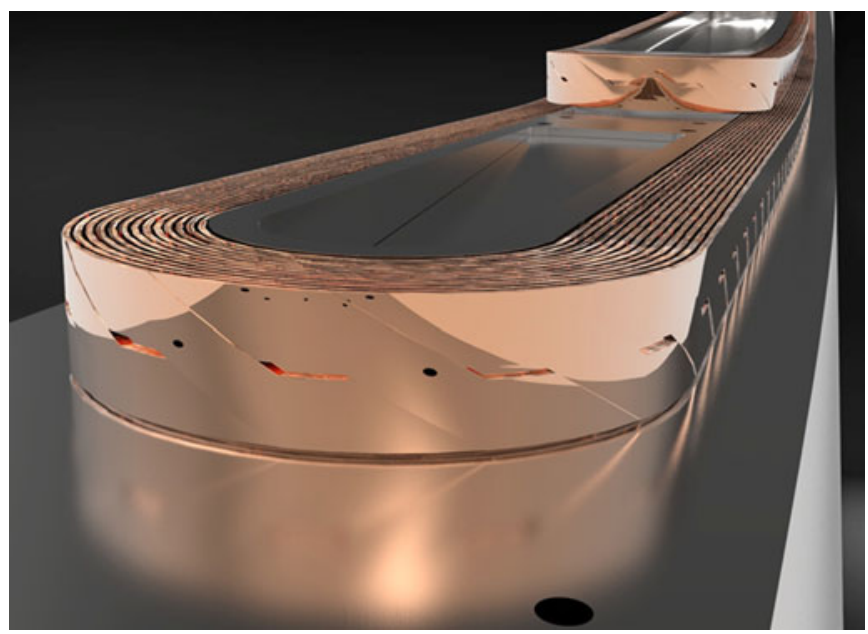

Fig. 1. This is a rendered image of the full three-dimensional Feather-M2 coil model. That was used during quench and field quality simulations. It contains the full geometry of the 15 tapes that form the REBCO cable that is wound into final coil.

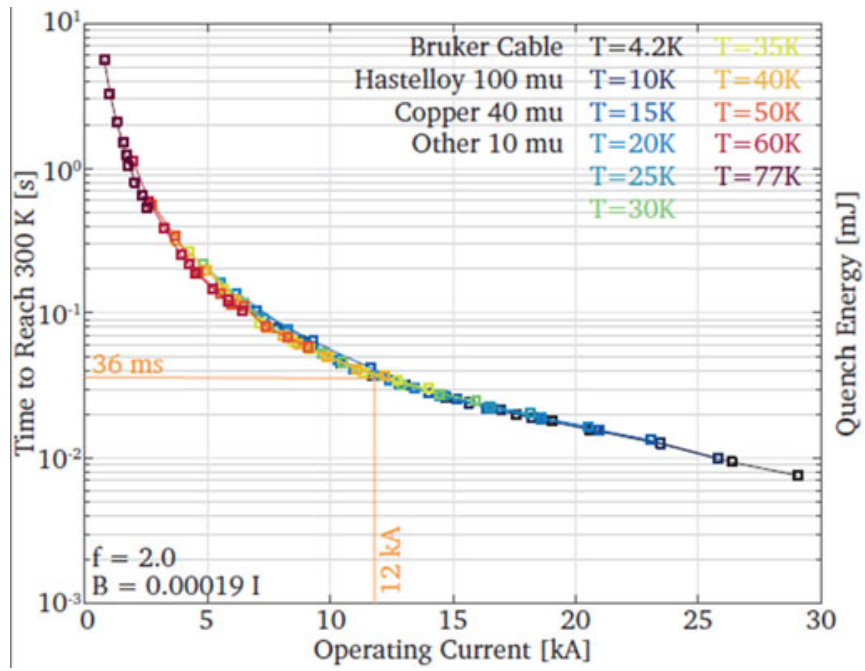

Fig. 2. Reaction time to $300 \mathrm{~K}$ as a function of magnet current and temperature calculated for the performance expected in the first Bruker tapes assembled into a Robel cable with the field in Feather-M0.4. A 3 turn coil.

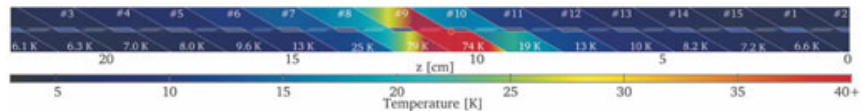

Fig. 3. Simulation showing thermal map of quenching Roebel cable.

code used for the HTS Roebel cable. Simulations and code descriptions can be found in [6]-[8].

\section{A. Quench Analysis With Simulated Signals}

The time of the "pre-quench drift" from nominal conditions to current sharing is very difficult to predict, because of complex structure and of strong anisotropy. A quench starts by forcing the edge of one tape over the critical surface. This spreads across that tape (see Fig. 3). Current starts to redistribute between

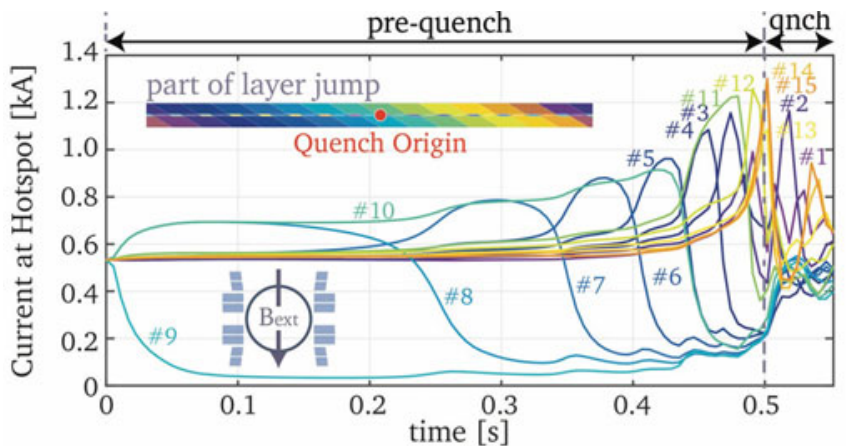

Fig. 4. Simulation of current redistribution between tapes in a cable leading to a full cable quench, approximately $400 \mathrm{~ms}$ after the start of current redistribution.

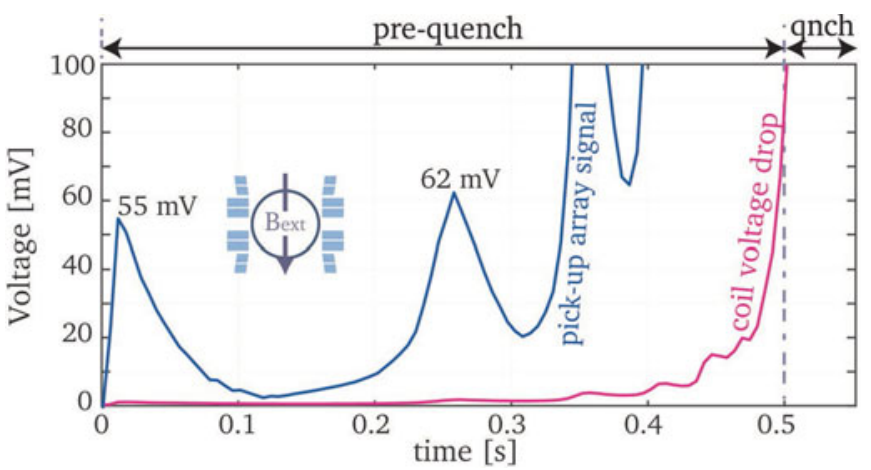

Fig. 5. Simulation of quench voltages in multi strand quenching cable. Red trace shows coil voltage, blue trace shows pick-up array voltage signals.

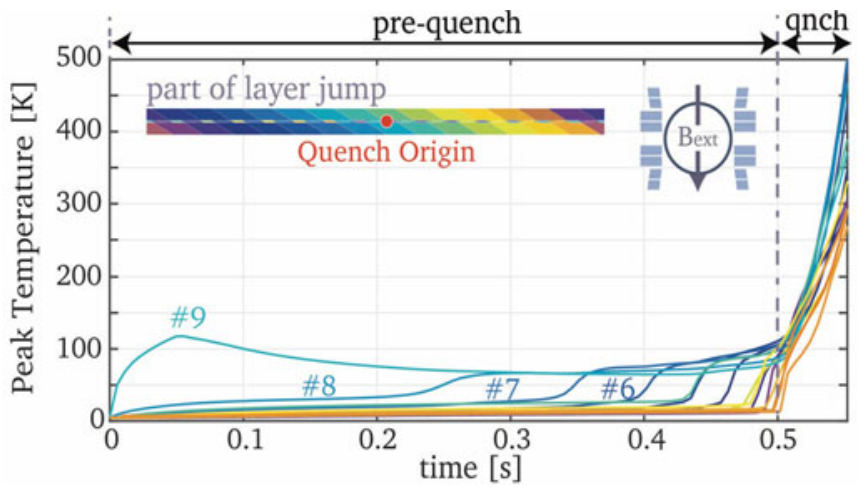

Fig. 6. Simulation of quench temperatures in multi strand quenching cable.

adjacent tapes. As the current exits the initial quenching tape, we see that its temperature is gradually reducing while other tapes in the cable begin to exceed the superconducting surface (see Figs. 3 to 6 which refer to the same quenching event). The voltages developed during this initial stage are extremely small, approximately $1 \mathrm{mV}$ (see Fig. 5 red trace). At this low voltage level noise may obscure the quench signals. As the last tapes in the cable quench, only then does the voltage grow rapidly at the same time as the temperature, leaving little time to react before a temperature run-away is initiated (see Figs. 5 and 6).

At high operating temperatures where the maximum available current density is lower, we have more time to react we therefore 


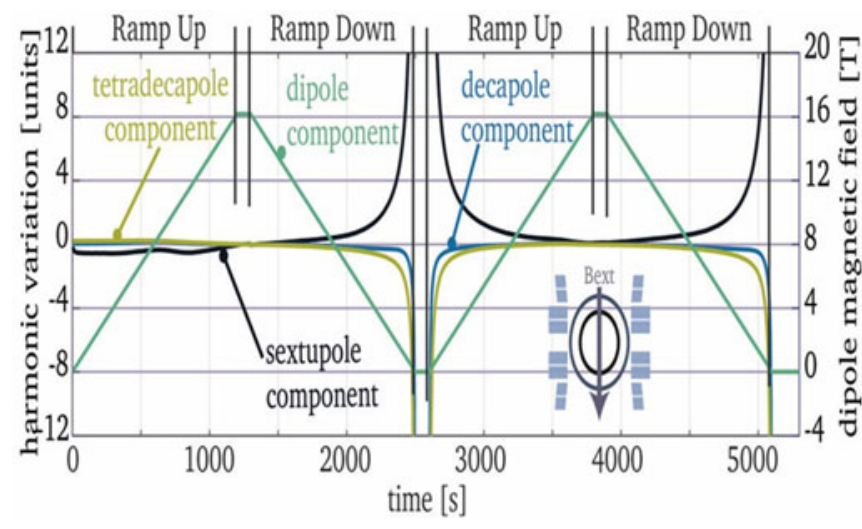

Fig. 7. Dynamic harmonics variation during ramping for Aligned Block design with cable oriented parallel to field lines, 10 units variation during two current ramps from 0 to $8000 \mathrm{~A}$ and back to $0 \mathrm{~A}$, in $13 \mathrm{~T}$ background field.
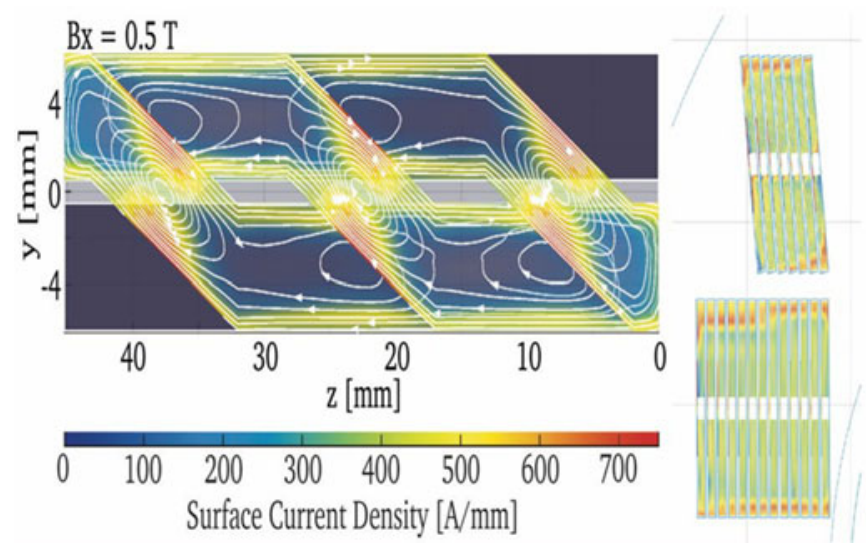

Fig. 8. (Left) cable current density looking at cable wide face. (Right) current density in tapes of cable, looking at a cut through the left side of FeatherM2 coil.

designed a test plan that would start at higher temperatures (see Fig. 2) and Section V, sub-section C, below.

The pre-quench drift should give an early indication that the quench is starting, (see Figs. 4 to 6), despite the very low voltages, was the idea behind the advanced quench detection systems which try to use the temperature or fields generated during the current redistribution within the cable. Temperature sensors placed throughout the coil proved to be impractical. However placing temperature sensors adjacent to the superconducting joints may be effective. Pickup coils are extremely promising (see Fig. 5 blue trace) and their design is described later in this paper. The quenching tape, locally redistributes current within the cable, this new current distribution can spread axially approximately $30 \mathrm{~m}$ along the cable. This leads to the possibility that a set of compact pick-up coil arrays, may be able to detect quenches up to $30 \mathrm{~m}$ from the quench origin.

\section{B. Field Quality}

Fig. 7 presents the dynamic field quality that was calculated for the Feather-M2 coil, when it is ramped in a background field of 13 T. In Fig. 8, we see the current distribution through the cable and coil after the current has been ramped up to $8000 \mathrm{~A}$ in

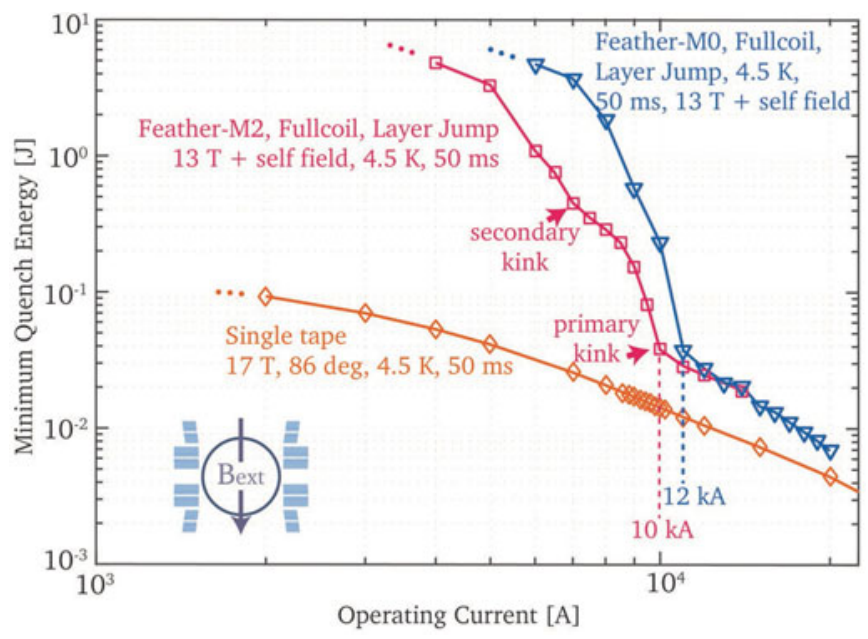

Fig. 9. Minimum quench energy calculations for a range of cables having different numbers of tapes in the cable at $4.2 \mathrm{~K}, \mathrm{Bo}=17 \mathrm{~T}$, á $=2^{\circ}$.

20 minutes similar to a LHC ramp profile. The light green areas in coil cross section image contain virtually no current. The red areas have high current density. The position of these currents will impose the final field quality, which is plotted in Fig. 7.

The field variation is very low under, 10 units. This calculation does not take into account iron saturation. The conclusion is that the variation in field errors due to current distribution within the coil appears to be controllable. The calculations for the Cosine Theta (CT) design exhibits higher errors in the range of 40 units due to the field angle at the magnet mid-plane, however much better than initially expectations.

\section{Power Losses Due to Magnetization}

The cable angle in relation to the field vector plays a strong role in the magnetisation losses. The Aligned Block (AB) design dissipates approximately 2 orders-of-magnitude less power dissipation than the (CT) design. The $\mathrm{AB}$ dissipates a maximum total power of $0.25 \mathrm{~W} / \mathrm{m}$ and Average $0.024 \mathrm{~W} / \mathrm{m}$. The CT dissipates a maximum total power of $24.4 \mathrm{~W} / \mathrm{m}$, with an average of $8.37 \mathrm{~W} / \mathrm{m}$ [9].

\section{Minimum Quench Energy in HTS Cable}

The minimum quench energy (MQE) for the HTS cable is in the range of Joules, approximately 3 orders-of-magnitude higher than the $\mathrm{Nb}_{3} \mathrm{Sn}$ and $\mathrm{Nb}$-Ti low temperature superconductors. When operating at lower temperature, $4 \mathrm{~K}$, the temperature margin can be as high as $50 \mathrm{~K}$. Therefore quenches in an operating magnet may only occur due to: heating due to cryogenic systems fail, catastrophic conductor failure through fatigue, temperature rise due to resistive joints, beam loss heating, or over-current induced during a fast ramp down of the outsert magnet. Coil movement leading to frictional heating, which have always been suspected for low temperature superconductor (LTS) quench initiation may not release sufficient energy to induce a quench. Fig. 9, presents the minimum quench energies for a selection of cables with varying numbers of tapes, starting 


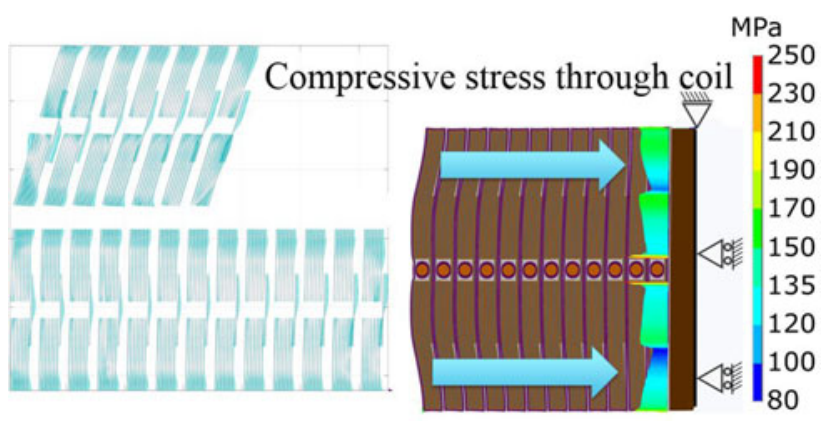

Fig. 10. (Left) image of pressure vectors in each tape of each cable in coil. (Right) horizontal pressure distribution in outermost single cable. Coil image shows high-pressure $>200 \mathrm{MPa}$ at the edges of the tapes. This case is calculated with a $17 \mathrm{~T}$ central field and perfect tape alignment.

with a single tape, moving up to the full 15 tape cable in the full FeatherM2 coil, in $13 \mathrm{~T}$ and self-field.

\section{E. Mechanical Analysis Coil Stress}

Knowing the precise position of the current flowing in individual tapes, it is then possible to calculate with finite element model (FEA) the build-up of stress through the coil pack. As the current predominantly flows at the edge of the tapes in the cable, the pressure generated due to the magnetic forces are also applied at the cable edges (see Fig. 10). The pressure is then calculated with a sophisticated FEA model, taking into account the full detail of impregnated glass sleeve around cable and sliding faces between cable tapes/strands. This predicted stresses can be locally as high as $200 \mathrm{MPa}$ compressive when inside the $13 \mathrm{~T}$ background field [10]. The University of Twente have just repeated their benchmark test, which applied a uniform pressure during powering of a short length of cable. The cable was insulated with the selected Feather-M2 impregnation system. No degradation up to $450 \mathrm{MPa}$ was seen [11]. The stress build up through the coil is diluted by shear stresses which are averaging out the peak stress. This highlights the need for detailed cable pressure measurement for further investigation and possible instrumentation during high field testing.

\section{III. “FeATHER-M2” ThE FIVE TeSLA MAgnet CONSTRUCTION STATUS Highlights}

\section{A. Feather-M2 Magnet Design Key Features}

Pickup coil arrays are placed on the magnet former. Then the insulated Robel cable is wound on to the former which has been treated with a mold release. Copper spaces pack out the volume between the coil and the energy extraction copper rings allowing adjustment for a range of cable thicknesses. Instrumentation is mounted. The coil assembly is then resin impregnated. Finally the two coils that make up the Feather-M2 magnet are mounted inside a High-strength tube. Joints are soldered and mechanically/electrically finished.

\section{B. 3D Printing Magnet Former}

Some features of the magnet former are "impossible" to machine conventionally so an additive manufacture approach was

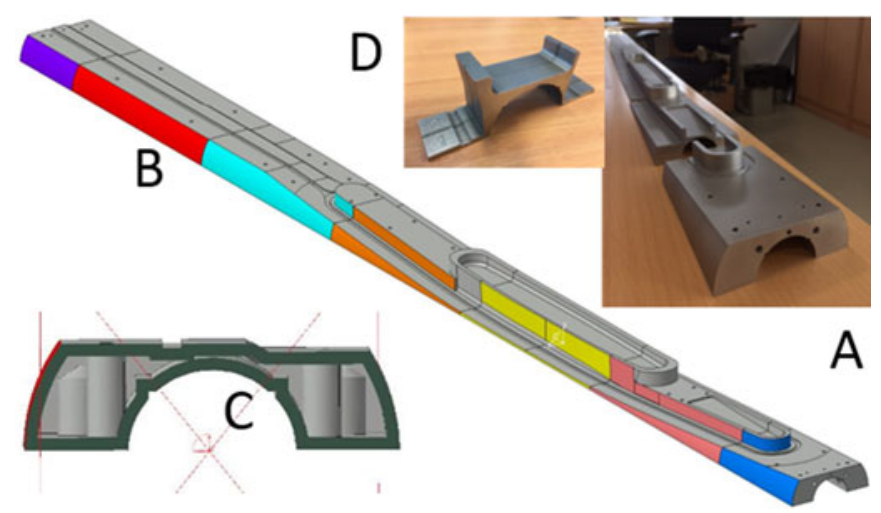

Fig. 11. Coil former produced with additive manufacture in 316L stainless steel. (A) 7-part Set of $212 \mathrm{~mm}$ long printed former sections, (B) former assembly model, (C) hollowed section III mm wall thickness for unloaded volumes, (D) laser welding test sample.

developed. The $1.3 \mathrm{~m}$ long former was too long to be printed in one piece, so an assembly of seven sections was needed, see Fig. 11.

The cost of the former is proportional to the volume of material. By hollowing out the former at non-loaded volumes the cost was reduced by $38 \%$. A magnet former wall thickness of 2.5 to $3 \mathrm{~mm}$ is an optimum for this component 3D printing, build stability. The former design was optimised through close collaboration with 3T in Newbury UK [12]. A Standard Material specification for Stainless steel 316L yield stress is $260 \mathrm{MPa}$. It is interesting to note that the 3-D printed material has a much improved yield stress of 470 - $530 \mathrm{MPa}$ depending on the build direction (vertical, horizontal, respectively). Laser welding would provide minimal distortion, but was not compatible with the micro-structure of the $3 \mathrm{D}$ printed surface. So before welding the contact surface must be machined.

\section{Pickup Coil Array}

The idea behind the pickup coil is to detect the flux change as one tape in the cable quenches and the current in that individual tape moves to the adjacent tapes (see Fig. 5). The changing flux position then induces a signal on the pickup coils. The pickup coils are photographically printed onto both sides of a $50 \mu \mathrm{m}$ Kapton sheet. Each coil has 285 full turns. There are 6 coils on the upper deck, and 24 coils on the lower deck. The copper coil pick-up tracks are 5 microns thick, 100 microns wide. The insulation space between tracks are 75 microns. The detailed design layout is presented in Fig. 12.

Polarity of the coils are chosen to match the twist pitch of the Robel cable. This amplifies the pickup signal. A flexible flat printed circuit board is electrically connected to the pick-up coil array to take the signal wires out of the magnet. During a full quench, low mutual inductance with the main field and high circuit impedance limits the pick-up coil currents.

\section{Joints}

Two joint types were required: first to connect the 15 tape Roebel cable to the cryostat current lead, and second, make the 


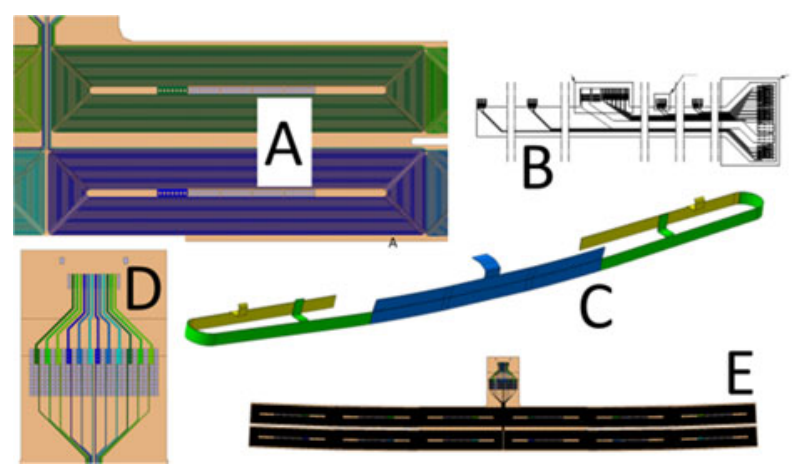

Fig. 12. Pickup coil array design details. (A) Rectangular pickup coil, (B) Exit flex-PCB trace, (C) full pickup coil array for one pole, (D) Printed circuit board connections between the five sheets, (E) Central 12 coil set.

connection between two Roebel cables which is the connection between the two poles of Feather-M2. A controlled solder procedure was performed using: low pressure (6 to $10 \mathrm{MPa})$, following the eutectic specific temperature profile, with short time at maximum temperature. The removable connection needed for the cryostat current lead used the LTS system. Were Roebel cable is soldered into a $300 \mathrm{~mm}$ long block (which is the final design length of the Roebel cable twist pitch), with a protective copper cover. This block is then lightly clamped to the current lead with an indium sheet. This allows the joint to be disassembled without delamination on the tapes in the cable. The $300 \mathrm{~mm}$ joint design will need to be improved as demonstrated when the joint voltage was exceeded in the powering test, descried later in Section IV, sub-section C, later.

\section{Feather-M0.4: Cable Performance \& INSTRUMENTATION}

\section{A. First REBCO Roebel Cable in Coil.}

The $12 \mathrm{~mm}$ wide Bruker tape was fine-blanked from a tape width of $5.5 \mathrm{~mm}$ and then silver coated, then copper coated with a $20 \mu \mathrm{m}$ layer. The 15 strand/tape Roebel cable that was assembled by KIT, has a transposition pitch of $226 \mathrm{~mm}$. This early copper coated tape exhibited "dog-bone" edges. Later, the "dog-boning" was improved. The predicted cable performance, when wound into the first test coil is presented in [13] \& [14]. This cable was then used to wound the first superconducting 3 turn subscale coil, named Feather-M0.4.

\section{B. Feather-M0.4 Instrumentation:}

As detailed in Fig. 13 the first Feather-M0.4 test coil contained the following instrumentation: voltage taps, pickup coils, temperature sensor array, calibrated point temperature sensors, Hall probes, integrating field coil and three spot heaters. The cryostat is equipped with thermocouples, CERNOX temperature sensors and many voltage taps.

\section{Cold Test, of Subscale CoIL Feather-M0.4}

\section{A. Variable Temperature Cooling System}

Liquid helium from a liquefier at $4 \mathrm{~K}$ is supplied to a 1001 satellite vessel. Its level is maintained at $50 \%$. 2-300 W heaters
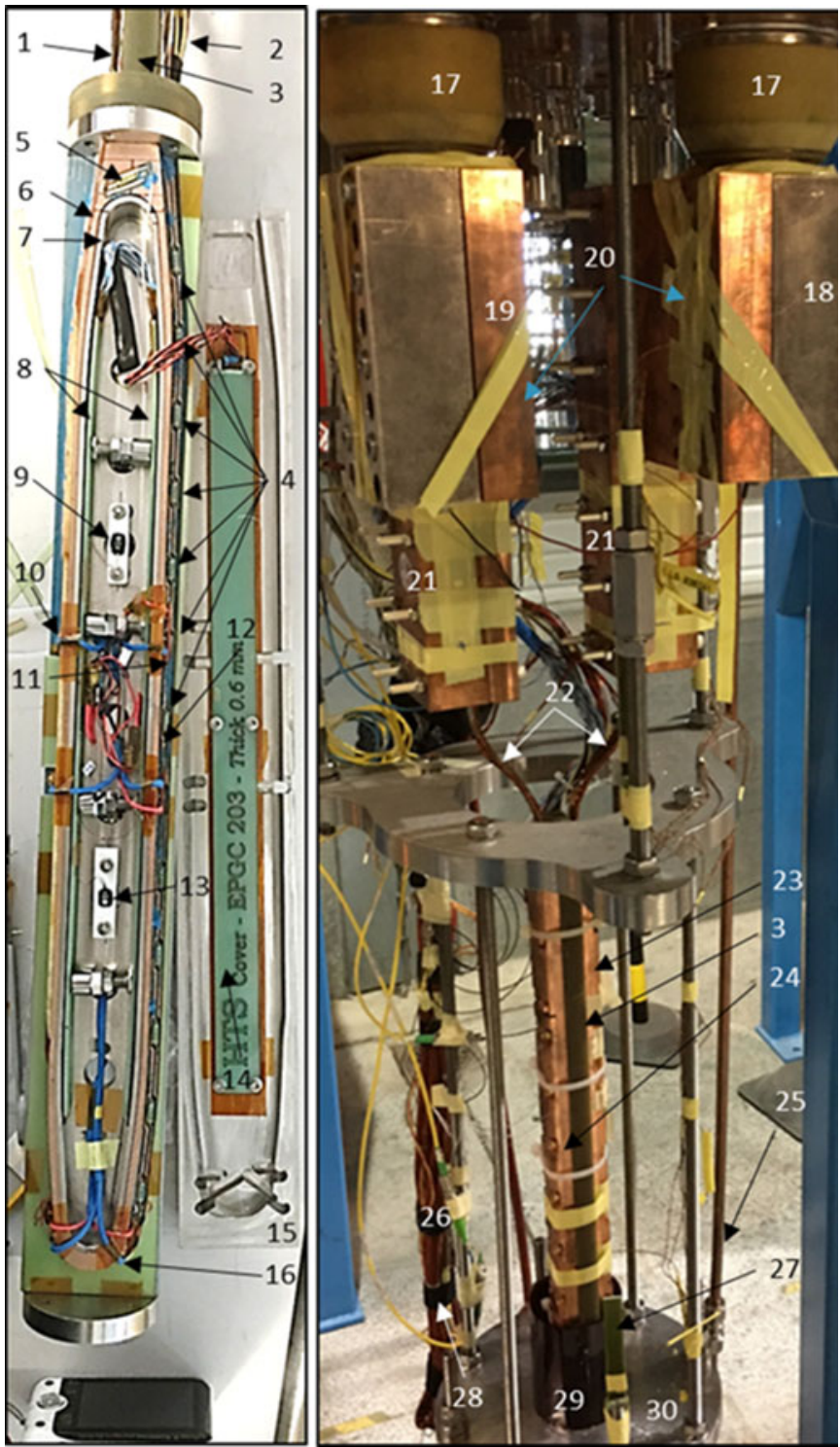

Fig. 13. (Left) Feather-M0.4 instrumented coil. (Right) Connections to test insert. Key: 1) Coil entry lead. 2) Coil exit lead, 3) Insulation between leads, seen in both pictures. 4) Part of CCS. Temp. Sensor array. 5) CCS temp sensor. 6) $\mathrm{Cu}$ coil former loop. 7) Magnet former support. 8) Pick-up coil sets. 9) Hall probe (a). 10) Spot heater \#1. 11) Spot heater \#2. 12) Position of fibre optic temp sensor in coil, adjacent to (11). 13) Hall probe (b). 14) Integrating field PCB coil. 15) Cover plate. 16) Spot heater \#3. 17) $15 \mathrm{kA}$ current leads. 18) -ve pole indium clamped joint. 19) + ve pole indium clamped joint. 20) Copper cryostat current lead to Roebel clamped connection adaptor plate. 21) Roebel cable clamped joints using indium sheets, Brass bolts. 22) Roebel cable link between Feather-M0.4 coil joint and cryostat current leads. With copper shunt \& Kapton insulation 23) Coil joint box -ve pole. 24) Coil joint box + ve pole. 25 ) Variable temp gas supply pipe to bottom of magnet. 26) Fibre optic connector 27) CERNOX temp sensor. 28) 4K helium auxiliary supply tube hidden in wires. 29) Kapton sheet ground insulation over Feather-M0.4 assembly that is inside the magnetic yoke. 30) Magnetic yoke top end plate.

are used to evaporate $>3 \mathrm{~g} / \mathrm{s}$ of cold gas to a second chamber containing a high performance variable power heater $500 \mathrm{~W}$ to 5 $\mathrm{kW}$, this sets the gas temperature in the $20 \mathrm{~K}$ to $300 \mathrm{~K}$ range with a stability better than $1 \mathrm{~K}$. A valve system splits the GHe gas flow and directs gas to the test cryostat and refrigerator return line. This enables variable mass flow delivery to cryostat with stable temperature control when low flow rates are required. A second $4 \mathrm{~K}$ helium liquid line is available passing into the 
magnet test cryostat for additional cooling of the current leads or other (see Fig. 13, item 28).

\section{B. HTS Quench Detection-Protection System}

Data acquisition is performed by three systems in parallel. The standard LTS test stand system, cRIO-9068 [15] and Micron Optics SM130 [16]. The test stand consists of a digital multimeter and a 'quench DAQ'. The digital multi-meter has the purpose of continuous temperature acquisition during cryostat operation with low frequency $(1 \mathrm{~Hz})$. The 'quench DAQ' stores its data in a certain time window when triggered (archive mode) at three different frequencies: $\mathrm{LF} 10 \mathrm{~Hz}, \mathrm{MF} 5 \mathrm{kHz}$ and $\mathrm{HF}$ $200 \mathrm{kHz}$. The cRIO is fitted with 7 NI-9205 [17] modules, with 32 channels and a maximum aggregate sample frequency of $250 \mathrm{kHz}$. Pick up coils and hall probes are measured with $10 \mathrm{kHz}$, while temperature sensors and system state signals are measured with $100 \mathrm{~Hz}$. A NI 9401 DO-module [18] is used for synchronisation, protection triggering and provoked quench heater control. Synchronisation triggers are send to the two other DAQ systems for data-fitting. Heaters are switched by solid state relays with power is delivered by a GEN3300W. Voltage and current is measured by the cRIO for quench energy estimations. Quench detection is performed by the PotAim and the cRIO in parallel. The PotAim processes all quench related data with respect to the SM18 system and the voltage taps in Feather-M0. Quench detection of all other signals coming from the magnet is performed by the cRIO. Both systems use a comparison of signals with a threshold over a predefined time window. Protection trigger of the cRIO is directly fed into the CERN SM18 test station safety matrix.

\section{Summary of Initial Cold Testing}

Roebel cable to current lead joint testing started in March 2016. The circuit was successfully powered to $5 \mathrm{kA}$, the temporary room temperature current lead limit. Cold testing of Feather-M0.4 coil started June 2016. Initially cooling to $4 \mathrm{~K}$ liquid maintaining a $20 \mathrm{~K}$ temperature difference over the magnet at $2.4 \mathrm{~K} / \mathrm{min}$ was achieved. We did not see a sharp transition to the superconducting state, so it was difficult to say at which temperature the coil becomes superconducting. This also is true for the Coil RRR 5. Using a 600 amp power supply low current powering was performed so that these sensor system noise could be assessed, leading to some rewiring and removal of unwanted additional Earth connections. There are three significant joint types within Feather-M0.4 assembly: the Copper Indium joint to the bottom of the cryostat current lead (see Fig. 13, items 18 \& 19), (approximately $170 \mathrm{n} \Omega @ 70 \mathrm{~K}$ and $100 \mathrm{n} \Omega$ @ $50 \mathrm{~K}$ ), Roebel cable to copper transition plate (see Fig. 13, item 21), $(<10 \mathrm{n} \Omega)$, and the Roebel cable to Roebel cable joints (see Fig. 13, items $23 \& 24),(<10 \mathrm{n} \Omega)$. Joint resistances were measured up to $3000 \mathrm{~A}$, at $56 \mathrm{~K}$. Coil inductance was measured to be $6 \mu \mathrm{H}$, in line with calculations, an extremely low value. The Test plan aimed to set high temperatures $80 \mathrm{~K}$ to $85 \mathrm{~K}$ where energy and cable Ic are low (see Fig. 14). Using spot heaters followed by power supply current extraction after a safe set time interval (See Fig. 6) we could fine tune the FPGA program to detect

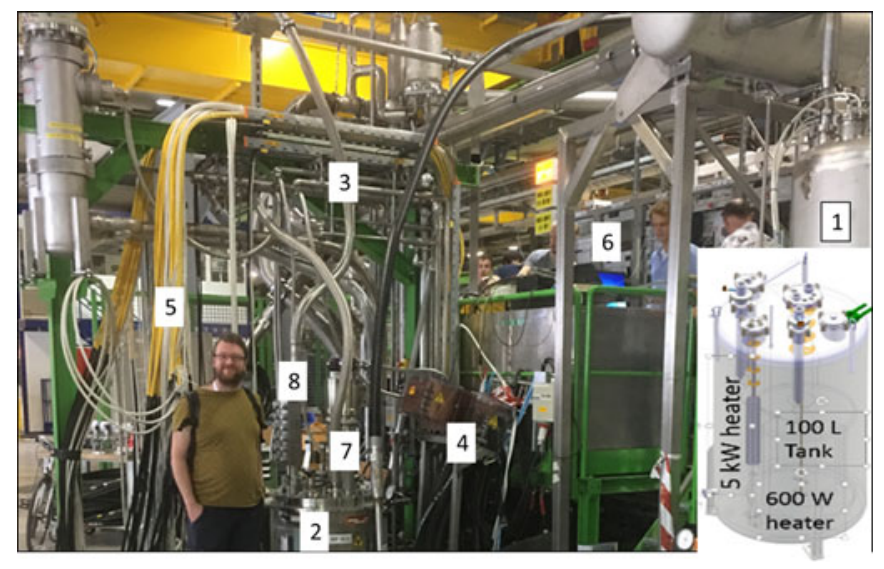

Fig. 14. The CERN test station "SM18", project students, \& SM18 test team. Key: 1) Variable temperature gas supply cryostat. 2) Magnet test cryostat. 3) Variable temperature Helium gas supply line. 4) Connection to the $20 \mathrm{kA}$ power supply through water $15 \mathrm{kA}$ cooled leads. 5) Instrumentation shielded cables between magnet and protection system. 6) Instrumentation electronics racks. 7) 15 kA current leads. 8) Fisher instrumentation connector box.

quenches and map short sample down to approximately $60 \mathrm{~K}$ the limit of the current lead capacity. The goal of this test was to commissioning of the advanced quench detection system, to be sure we can rely on it for Feather-M2 protection. Temperature stability at the higher temperatures was very good, less than $1 \mathrm{~K}$. Temperature difference between the satellite vessel and the test cryostat with the HTS Feather-M0.4 coil was approximately $5 \mathrm{~K}$. After a thermal cycle to room temperature, the $20 \mathrm{kA}$ power supply was connected. Working at higher temperatures than normal care was taken with the $12 \mathrm{kA}$ LTS current leads. Some adjustment was needed to balance the circuit as the control system gave an unusual current oscillation at the point of switching on, because of the very small inductance and almost negligible resistance, as the power supply was turned on the current sat at 20 A then jumped to $600 \mathrm{~A}$, followed by what seemed to be a controlled ramp down during several minutes to $80 \mathrm{~A}$. After this the current controlled within milliamps. At 1700 A we triggered the energy extraction with an $80 \mathrm{mOhm}$ resistor. After an initial 400 A over $1 \mathrm{~ms}$ increase in current, the current was extracted in a total of $4 \mathrm{~ms}$ which is important for future protection planning. During joint resistance measurements which follow an up and down current staircase powering profile. At $2600 \mathrm{~A}$ and $60 \mathrm{~K}$, we saw the voltage across joint 2 slowly rising (Black curve in the plot of Fig. 15). The current was manually driven down. Running the current back up to 2600 A taking careful note of voltage, the voltage increase was reproduced. The Helium mass flow rate was then increased from $1.5 \mathrm{~g} / \mathrm{s}$ to $3 \mathrm{~g} / \mathrm{s}$ and we saw a small voltage reduction while maintaining the same temperature and current. Further tests with reduced temperature $56 \mathrm{~K}$ saw the joint-2 voltage start to runaway at $3000 \mathrm{~A}$ (see Fig. 15). Coil was powered to $3200 \mathrm{~A}$ for short periods. The joint-2 voltage was limiting our ability to quench the magnet in the coil. After adding additional cooling to all joints, the magnet was powered to quench over a range of gradually reducing temperatures. The joint temperatures were always approximately $20 \mathrm{~K}$ lower than the magnet. In Fig. 16 we see a selection of joint trips and coil 


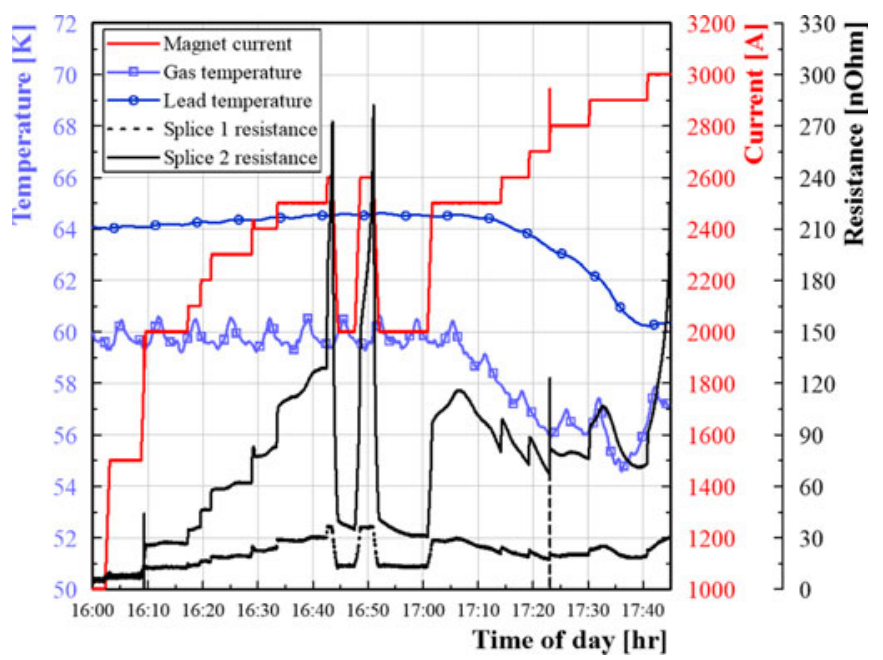

Fig. 15. Test day 3, Feather0-4 test plot showing increase in joint-2 resistance during powering. Temperature stability, joint resistance as temperature is decreased and current increased.

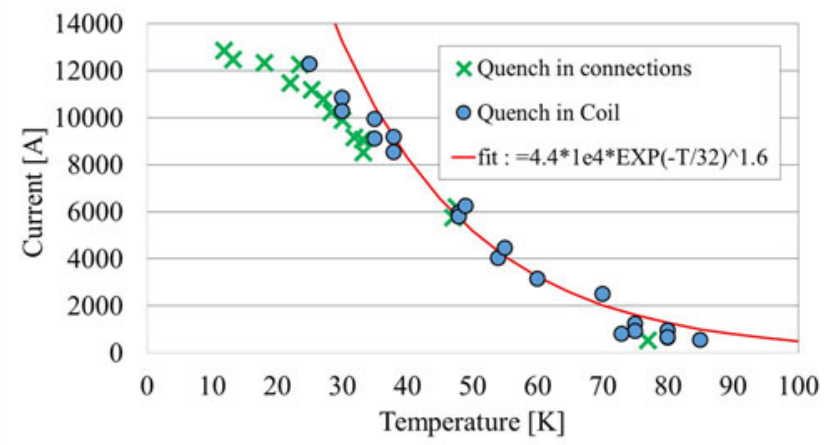

Fig. 16. Feather M0.4 cold test quenches v temperature plot. We see a selection of coil quenches and joint voltage trips.

quenches. A maximum current of just over $12.9 \mathrm{kA}$ was reached in $25 \mathrm{~K}$ helium gas, when a joint voltage tripped the protection. This exceeded our target current value $10 \mathrm{kA}$. At $13 \mathrm{~K}$ the cryostat started to fill with liquid helium. The test was then stopped, due to the uncertainty in being able to operate safely in liquid helium. Further testing is planned.

\section{DISCUSSION AND CONCLUSION}

Developing high current cables, coils, joints and quench protection for the HTS high current magnets is a highly demanding challenge and has advanced greatly with the EuCARD2 HTS magnet program. The recent success in being able to simulate current distribution and quench in the multi-strand Roebel cable is fundamental to all tape based, good field quality magnet developments. The potential to use innovative quench detection systems, such as pick-up coil arrays, or temperature sensors in liquid Helium is promising but still needs to be proven. The first high current HTS coil, Feather-M0.4 containing Bruker Tape and KIT Roebel cable has been powered above $12.9 \mathrm{kA}$ in $25 \mathrm{~K}$ gas and quenched over 100 times without degrading the coil. This is a significant milestone. The next Feather-M0 coils, exploring different features have started to be produced. The first full-scale 5 T Feather-M2.1 Aligned Block design and its alternative, CEA Saclay Paris, Cosine Theta coil are both expected to be cold tested in spring 2017. The technical challenges that we are still facing are: detecting quenches with the magnet submerged fully in liquid helium, the high current HTS joint design and operation, operating in high external magnetic field and finally controlling magnetic field-quality using the $5 \mathrm{~mm}$ wide tapes.

\section{REFERENCES}

[1] F. Bordry, S. Baird, K. Foraz, A. L. Perrot, R. Saban, and J.P Tock, "The first long shutdown (LS1) for the LHC," in Proc. Int. Particle Accelerator Conf., 2013, pp. 44-48. [Online]. Available: http://accelconf. web.cern.ch/AccelConf/IPAC2013/papers/mozb202.pdf

[2] L. Rossi, "LHC upgrade plans: Options and strategy," in Proc. Int. Particle Accelerator Conf., San Sebastian, Spain, 2011, pp. 908-912. [Online]. Available: http://accelconf.web.cern.ch/AccelConf/ IPAC2011/papers/tuya02.pdf

[3] L. Bottura, G. de Rijk, L. Rossi, and E. Todesco, "Advanced accelerator magnets for upgrading the LHC," IEEE Trans. Appl. Supercond., vol. 22, no. 3, Jun. 2012, Art. no. 4002008.

[4] L. Rossi et al., "The EuCARD-2 future magnets European collaboration for accelerator-quality HTS magnets," IEEE Trans. Appl. Supercond. vol. 25, no. 3, Jun. 2015, Art. no. 4001007.

[5] G. A. Kirby et al., "Accelerator quality HTS dipole magnet demonstrator designs for the EuCARD-2, 5 tesla $40 \mathrm{~mm}$ clear aperture magnet," IEEE Trans. Appl. Supercond., vol. 25, no. 3, Jun. 2015, Art. no. 4000805.

[6] G. Kirby et al., "Status of the demonstrator magnets for the EuCARD-2 future magnets project," IEEE Trans. Appl. Supercond., vol. 26, no. 3, Apr. 2016, Art. no. 4003307.

[7] J. Van Nugteren et al., "Measurement and numerical evaluation of ACloss in a ReBCO roebel cable at $4.5 \mathrm{~K}$," IEEE Trans. Appl. Supercond., vol. 26, no. 3, Apr. 2016, Art. no. 8201407.

[8] J. Van Nugteren, "High temperature superconductor accelerator magnets," Ph.D. dissertation, University of Twente, Enschede, The Netherlands, 2016.

[9] J. Van Nugteren et al., "Numerical analysis of magnetisation in coated conductor cables and coils," in Proc. Appl. Supercond. Conf., 2006, Paper 1SPOr1A-06.

[10] J. Murtomäki et al., "Mechanical effects of the non-uniform current distribution on HTS coils for accelerators wound with ReBCO Roebel cable," in Proc. Appl. Supercond. Conf., 2006, Paper 3LPo2F-03.

[11] P. Gao et al., "Effect of tape layout and impregnation method on the transverse pressure dependence of the critical current in REBCO Roebel cables," in Proc. Appl. Supercond. Conf., 2006, Paper 2LPo2D-06.

[12] 3T, Additive manufacturer's website. [online]. Available: http://www.3trpd.co.uk/.

[13] W Goldacker et al., "Roebel cables from REBCO coated conductors: A one-century-old concept for the superconductivity of the future," Supercond. Sci. Technol., vol. 27, 2014, Art. no. 093001.

[14] C. Lorin et al., "Development of a Robel-cable-based Cos $\vartheta$ dipole: design and wind ability of magnet ends," presented at Eur. Conf. Appl. Supercond. (EUCAS), 2015 Lyon, France.

[15] cRIO-9068 FPGA system NI 9068. [Online]. Available: http://sine.ni.com/ nips/cds/view/p/lang/en/nid/211620

[16] Micron Optics sm130. [Online]. Available: http://www.micronoptics.com/ product/dynamic-optical-sensing-interrogator-sm130/

[17] NI 9205. [Online]. Available: http://sine.ni.com/nips/cds/view/p/lang/en/ nid/208800

[18] NI 9401. [Online]. Available: http://sine.ni.com/nips/cds/view/p/lang/en/ $\mathrm{nid} / 208809$ 